\title{
The Role of the Mesenchyme in Mouse Neural Fold Elevation II. Patterns of Hyaluronate Synthesis and Distribution in Embryos Developing In Vitro
}

\author{
JOYCE MORRIS-WIMAN AND LINDA L. BRINKLEY \\ Department of Anatomy \& Cell Biology, The University of Michigan Medical School, Ann Arbor, MI 48109
}

\begin{abstract}
Hyaluronate (HA) distribution patterns were examined in the cranial mesenchyme underlying the mesencephalic neural folds of mouse embryos maintained in roller tube culture. Using standard image-processing techniques, the digitized images of Alcian blue-stained or ${ }^{3} \mathrm{H}$-glucosamine-labeled sections digested with an enzyme specific for $\mathrm{HA}$, were subtracted from adjacent, undigested sections. The resultant difference picture images (DPI) accurately depicted the distribution of stained or labeled $\mathrm{HA}$ within the cranial mesenchyme. ${ }^{3} \mathrm{H}$-glucosamine-labeled HA was distributed uniformly throughout the cranial mesenchyme as 12, 18, and $24 \mathrm{hr}$ of culture. By contrast, the mesenchyme was uniformly stained with Alcian blue at $12 \mathrm{hr}$, but stain intensity decreased in the central regions of the mesenchyme at 18 and $24 \mathrm{hr}$.
\end{abstract}

HA distribution patterns were also examined in the cranial mesenchyme of embryos cultured in the presence of diazo-oxo-norleucine (DON), a glutamine analogue that inhibits glycosaminoglycan and glycoprotein synthesis. In DON-treated mesenchyme, Alcian blue staining of $\mathrm{HA}$ was decreased from that in controls at 12, 18, and $24 \mathrm{hr}$. However, incorporation of ${ }^{3} \mathrm{H}$-glucosamine into $H A$ was increased. The distribution of labeled HA within treated mesenchyme as 12,18 , and $24 \mathrm{hr}$ resembled that in controls at $12 \mathrm{hr}$.

These results indicate that the distribution of $H A$ within the cranial mesenchyme of normal mouse embryos during neural fold elevation and convergence is not determined solely by regional differences in HA synthesis. We propose that HA distribution patterns result from the expansion of the HA-rich extracellular matrix of the central mesenchyme regions. This expansion may play a major role in fold elevation. These results also suggest that DON treatment reversibly inhibits HA synthesis, since treated mesenchymal cells retain the capability of synthesizing $\mathrm{HA}$ when provided with a glucosamine substrate. Patterns of ${ }^{3} \mathrm{H}$-glucosamine incorporation by DON-treated mesenchyme are similar to those observed in control mesenchyme prior to mesenchymal expansion at $12 \mathrm{hr}$.

\section{INTRODUCTION}

Distinct temporal and spatial patterns of distribution of cells and extracellular matrix are observed in the cranial mesenchyme underlying the mouse mesencephalic neural folds (Morris-Wiman and Brinkley, 1990a). A decrease in cell density and hyaluronate (HA) concentration in the central regions of the cranial mesenchyme is correlated with increased cross-sectional area of the total mesenchymal compartment and with the elevation and convergence of the neural folds. In a companion study, the analysis of patterns of mesenchymal cell distribution and mitotic activity during neural fold elevation and convergence indicates that the decrease in mesenchymal cell density is not the result of a decrease in the mitotic rate or the cell-survival rate in the central mesenchyme (Morris-Wiman and Brinkley, 1990b). Alternatively, the decrease in cell density could be produced by the greater expansion of the mesenchyme in its more central regions relative to its expansion in other regions. The major extracellular component of the cranial mesenchyme of mammalian neural folds is the glycosaminoglycan, hyaluronate (Solursh and Morriss, 1977; Morriss and Solursh, 1978a,b; Heifetz et al., 1980). In other developmental systems, high concentrations of HA are associated with the sudden expansion of intercellular spaces that precedes cell migration or accompanies tissue remodeling (for review, see Toole, 1981). Prior to the expansion of the cranial mesenchyme, the HA content of the central regions of the mesenchyme increases (Morris-Wiman and Brinkley, 1990a). The expansion of the HA-rich extracellular matrix of the central mesenchyme would serve to displace and separate resident mesenchymal cells, decreasing their density, as well as to decrease the relative concentration of $\mathrm{HA}$ in this region.

A decreased synthesis or increased degradation of HA could also explain the decrease in HA content of the extracellular matrix of the central mesenchyme which accompanies expansion and elevation. In this study, to determine what role regional differences in synthetic rates play in establishing patterns of HA distribution during fold morphogenesis, we have analyzed ${ }^{3} \mathrm{H}$-glu-

Received April 25, 1989. Accepted January 3, 1990.

Address reprint requests to Joyce Morris-Wiman, Department of Orthodontics, University of Florida College of Medicine, Gainesville, FL 32610. 
cosamine-labeling and Alcian blue staining patterns in computer-generated difference picture images (DPI). DPIs are created using a computer method that permits subtraction of a ${ }^{3} \mathrm{H}$-glucosamine-labeled or Alcian-bluestained section from an adjacent section that has been digested with an enzyme that specifically degrades HA. The resultant DPI is an accurate representation of ${ }^{3} \mathrm{H}$ glucosamine-labeled or Alcian-blue-stained HA within the cranial mesenchyme. The results of analysis of DPIs indicate that $\mathrm{HA}$ synthetic rates do not predict patterns of HA distribution; thus the decrease in HA concentration in the central mesenchyme is not the result of regional differences in synthesis.

This study also analyzes ${ }^{3} \mathrm{H}$-glucosamine incorporation and HA distribution patterns in the cranial mesenchyme of embryos cultured in the presence of diazooxo-norleucine (DON), a glutamine analogue that inhibits the synthesis of glycosaminoglycans and glycoproteins from glucosamine. Our results indicate that, when given an exogenous source of glucosamine in ${ }^{3} \mathrm{H}$-glucosamine, cells in DON-treated mesenchyme synthesize glycosaminoglycans and glycoproteins. The distribution of ${ }^{3} \mathrm{H}$-glucosamine-labeled material is similar to that observed in control mesenchyme prior to mesenchymal expansion.

\section{MATERIALS AND METHODS Embryo Culture Conditions}

Embryos were cultured as described previously (Morris-Wiman Brinkley, 1990b) in the following media. Embryos for use in the analysis of ${ }^{3} \mathrm{H}$-glucosamine incorporation were cultured in $1.0 \mathrm{ml}$ of immediately centrifuged, heat-inactivated rat serum (Steele and New, 1974), $1.0 \mathrm{ml}$ of $\mathrm{BGJ}_{\mathrm{b}}$ medium (Fitton-Jackson modification), $0.05 \mathrm{ml}$ garamycin $(20 \mathrm{mg} / 100 \mathrm{ml})$ and $0.1 \mathrm{ml}$ of sterile water; embryos for ultrastructural analysis were cultured in $1.0 \mathrm{ml}$ rat serum, $0.5 \mathrm{ml} \mathrm{FC}$ 43 (O'Shea et al., 1982), $0.5 \mathrm{ml} \mathrm{BGJ}_{\mathrm{b}}$ medium (FittonJackson modification), $0.05 \mathrm{ml}$ garamycin $(20 \mathrm{mg} / 100$ $\mathrm{ml})$ and $0.1 \mathrm{ml}$ sterile water. Diazo-oxo-norleucine (DON) $(0.05 \mathrm{ml}, 2.0 \mu \mathrm{g} / \mathrm{ml}$, Sigma, St. Louis, MO) or sterile water was added to all cultures before the addition of the embryos.

${ }^{3} \mathrm{H}$-Glucosamine $(25 \mu \mathrm{Ci} / \mathrm{ml}$, sp. act. $38 \mathrm{Ci} / \mathrm{mmole}$; ICN, Irvine, CA) was added to the culture media at 4 , 10,16 , and $22 \mathrm{hr}$ of culture. The bottles were regassed after the addition of ${ }^{3} \mathrm{H}$-glucosamine or after $12 \mathrm{hr}$. Embryos were labeled for $2 \mathrm{hr}$ and then removed from culture. Embryos used for ultrastructural analysis were removed from culture after 8,16 , and $24 \mathrm{hr}$ of culture. Embryos were then processed as described below.

\section{Histological Techniques}

Embryos designated for use in the analysis of Alcian blue-staining and ${ }^{3} \mathrm{H}$-glucosamine-incorporation patterns were fixed for $4 \mathrm{hr}$ in $4 \%$ paraformaldehyde in $0.1 \mathrm{M}$ phosphate buffer $(\mathrm{pH} 7.2)$ with $0.5 \%$ cetylpyridi-

$\begin{array}{ll} & \\ \mathrm{d} & \text { dorsal aorta } \\ \mathrm{g} & \text { gut } \\ \mathrm{m} & \text { mesenchyme } \\ \mathrm{n} & \text { notochord } \\ \mathrm{NE} & \text { neuroepithelium } \\ \mathrm{s} & \text { surface ectoderm }\end{array}$

nium chloride (CPC) added to precipitate glycosaminoglycans (Derby and Pintar, 1978). They were then dehydrated in graded ethanols, cleared in methyl benzoate, and embedded in Paraplast. Frontal sections ( $7 \mu \mathrm{m})$ were cut and mounted, alternating between two sets of subbed microscope slides to permit comparison of patterns of Alcian blue staining with patterns of ${ }^{3} \mathrm{H}$-glucosamine incorporation. Consecutive sections were incubated in either $100 \mathrm{TRU} / \mathrm{ml}$ Streptomyces hyaluronidase (SH) (ICN, Irvine, CA) in $0.1 \mathrm{M}$ sodium acetate buffer ( $\mathrm{pH}$ 5.0) or in buffer alone. The slides containing sections designated for autoradiography were then dipped in Kodak NTB2 emulsion, diluted 1:1 with glass-distilled water, and exposed for 2 weeks at $4^{\circ} \mathrm{C}$. They were developed in D-19 for $4 \mathrm{~min}$, rinsed in distilled water, fixed in Kodak rapid fixer, and allowed to air-dry overnight. The slides containing sections designated for Alcian blue staining were rinsed briefly in $5 \mathrm{~N} \mathrm{HCl}$ to remove nuclear staining, and then stained overnight in 1\% Alcian blue (Mallinckrodt, Paris, KY; Lot KPPG) in $3 \%$ acetic acid ( $\mathrm{pH} 2.6$ ) with $0.025 \mathrm{M} \mathrm{MgCl}_{2}$. All sections were dehydrated in graded ethanols, cleared in xylene, and mounted in Permount.

Embryos for ultrastructural analysis were fixed in $1 \%$ glutaraldehyde in $0.1 \mathrm{M}$ phosphate buffer $(\mathrm{pH} 7.2$ ) with $2 \%$ tannic acid to retain glycosaminoglycans (Singley and Solursh, 1980) for $2 \mathrm{hr}$. They were postfixed in $1 \%$ osmium tetroxide in $0.1 \mathrm{M}$ phosphate buffer for $1 \mathrm{hr}$, dehydrated in graded ethanols, cleared in propylene oxide, and embedded in Epon. Silver lo gold sections were cut on a Huxley LKB ultramicrotome and stained with uranyl acetate and lead citrate. Sections were examined with a Phillips 400 EM.

\section{Image Processing}

Streptomyces hyaluronidase-digested and adjacent undigested Alcian blue-stained or ${ }^{3} \mathrm{H}$-glucosamine-labeled sections of the mesencephalic neural folds of four embryos were digitized from a Leitz Diaplan microscope with a DAGE Neuvicon camera. The visualization of Alcian blue stain was intensified by placing a $603+10 \mathrm{~nm}$ filter in the light path. The images were recorded as $512 \times 512$ pixel matrices of brightness values 0 (black) to 255 (white).

These images were analyzed using a computer-assisted method described previously (Brinkley and Morris-Wiman, 1987; Morris-Wiman and Brinkley, 1990a) and briefly reviewed here. Using an International Imaging Systems (IIS, San Jose, CA) image processor linked to a MassComp MC500 computer (Westford, MA), the digested and undigested images were registered and subtracted to produce an image with gray values representing only the stained or labelled material that was removed by SH-digestion, i.e., HA. In the autoradiographs, this image subtraction was preceded by preprocessing the images with a median filter of a 5 $\times 5$ matrix to eliminate separate silver grains and produce an image having a smooth range of brightness values. Because Paraplast sections expand unevenly when applied to subbed microscope slides, even consecutive sections do not register well enough to permit their subtraction. The IIS has an indwelling warper that was used to fit the digested image to the undigested. The two images were then registered and subtracted to produce a difference picture image (DPI). 
The DPIs, as well as the filtered images of SH-digested and undigested ${ }^{3} \mathrm{H}$-glucosamine-labeled sections, were mapped into a standard histogram with a median gray value of 128 and a standard deviation of 27.5. The background and cells of these images were found to have gray level values above 150. All gray values above 150 were mapped to 255 , or white, to produce an image containing only gray values that corresponded to either ${ }^{3} \mathrm{H}$-glucosamine or Alcian blue stain. These images were further processed using a thresholding function which segmented the range of gray values into six steps, black to white. Each step of increasing density represents an increase in the relative concentration of ${ }^{3} \mathrm{H}$-glucosamine or Alcian blue. To determine the percent area of the mesenchyme covered by grains attributable to ${ }^{3} \mathrm{H}$-glucosamine-labeled $\mathrm{HA}$, DPIs created from labeled Streptomyces hyaluronidasedigested and undigested sections were converted to binary images, images in which all gray levels intermediate between 1 and 255 were turned to black. The percent area of the mesenchyme covered by grains was computed from these images using available software on the IIS.

\section{RESULTS}

Distribution of Alcian Blue Stained Hyaluronate

In order to evaluate objectively the temporal and spatial distribution of hyaluronate (HA) in the cranial mesenchyme of the neural folds, we analyzed patterns of Alcian blue distribution in difference picture images (DPI). A DPI is the result of the subtraction of the image of a section digested with Streptomyces hyaluronidase $(\mathrm{SH})$, an enzyme that specifically degrades HA (Ohya and Kaneko, 1970), from the image of an adjacent, undigested section of the neural fold. The image produced, the DPI, should be an accurate depiction of the Alcian blue-stained HA within the cranial mesenchyme and neural fold.

\section{Controls}

In controls at $6 \mathrm{hr}$ of culture (Fig. 1), the mesenchyme adjacent to the median neural groove and dorsal to the dorsal aorta stains more intensely for HA than do lateral mesenchymal regions. This medial mesenchymal region retains its intense Alcian blue staining at $12 \mathrm{hr}$. However, the dorsal portions of the central and lateral mesenchyme also stain intensely, indicating an increase in HA concentration in these regions. At $18 \mathrm{hr}$, the relative amount of Alcian blue-stained HA decreases significantly in the central mesenchyme, whereas the intense staining of the dorsolateral and medial mesenchyme is maintained. The neural folds of embryos cultured for $24 \mathrm{hr}$ are characterized by a heavy stain deposition in the lateral mesenchyme. The relative amount of stain appears to be decreased in the central and medial regions, indicating a decrease in the $\mathrm{HA}$ concentration in these regions. At all culture times, the basement membrane of the median neural groove and adjacent neuroepithelial rim is more intensely stained than the basement membrane of the central and lateral fold neuroepithelium.

\section{DON-treated embryos}

Patterns of HA distribution in DON-treated and control mesenchyme are identical at $6 \mathrm{hr}$ of culture (Fig.
2). However, at $12 \mathrm{hr}$, the pattern of Alcian blue staining in DON-treated mesenchyme is distinctly different from that in controls. The treated mesenchyme stains less intensely for HA than does its control counterpart (cf. Figs. 1 and 2). Stain intensity is increased medially in the mesenchyme immediately adjacent to the median neural groove and notochord and also in the lateral mesenchyme enclosed by the junction of the neuroepithelium $(\mathrm{NE})$ and the surface ectoderm $(\mathrm{SE})$. The mesenchyme dorsal and medial to the dorsal aorta binds considerably less Alcian blue than in controls. This pattern of distribution is maintained in 18-hr treated mesenchyme. At $24 \mathrm{hr}$, the dorsomedial mesenchyme becomes more heavily stained. Staining may also be intensified in the region between the neuroepithelial basement membrane $(\mathrm{NE} / \mathrm{Bm})$ and subjacent enlarged blood vessels.

\section{Patterns of ${ }^{3} \mathrm{H}$-Glucosamine Incorporation into Extracellular Matrix Components}

Autoradiographs of the cranial mesenchyme of the mesencephalic neural folds labeled for $2 \mathrm{hr}$ in culture with ${ }^{3} \mathrm{H}$-glucosamine were analyzed to determine whether changes in the distribution of Alcian bluestained HA observed during neural fold elevation were the result of regional differences in HA synthesis. Patterns of total ${ }^{3} \mathrm{H}$-glucosamine incorporation, incorporation into Streptomyces-hyaluronidase-sensitive material (HA), and incorporation into Streptomyces-hyaluronidase-insensitive material (other GAG species and glycoproteins) were examined in the cranial mesenchyme of both control embryos and embryos cultured in the presence of DON.

\section{Patterns of Total ${ }^{3} \mathrm{H}$-Glucosamine Incorporation}

\section{Controls}

At $6 \mathrm{hr}$ of culture (Fig. 3), incubation with ${ }^{3} \mathrm{H}$-glucosamine results in the intense labeling of the cranial mesenchyme of the mesencephalic neural folds. Areas dorsal and lateral to the dorsal aorta are the most heavily labeled, whereas the medial mesenchyme adjacent to the median neural groove is relatively label free. Most of the label in the mesenchymal compartment is extracellular and is not associated with mesenchymal cells. By contrast, the neuroepithelial and surface ectodermal layers are labeled intracellularly. The extracellular material on the apical surfaces of these layers is also labeled. This is particularly true of the apical surfaces of the surface ectodermal cells enclosing the first arch. The basement membrane of the surface ectoderm (SE/Bm) of the first arch is also more heavily labeled than that of more dorsal regions. Label is densest in the $\mathrm{NE} / \mathrm{Bm}$ at the rim of the median neural groove and is indistinct at the neuroepithelial-surface ectoderm (NE/SE) junction.

The total amount of ${ }^{3} \mathrm{H}$-glucosamine incorporated into the extracellular matrix (ECM) of the mesenchyme is decreased at $12 \mathrm{hr}$ of culture, and the pattern of label distribution in the mesenchyme differs significantly from that observed at $6 \mathrm{hr}$ (Fig. 3). The dorsolateral mesenchyme at the base of the narrowed, elevating fold edge is now the most densely labeled region, whereas in the central and medial regions label intensity has diminished. The mesenchyme adjacent to the median neural groove remains relatively unlabeled as 

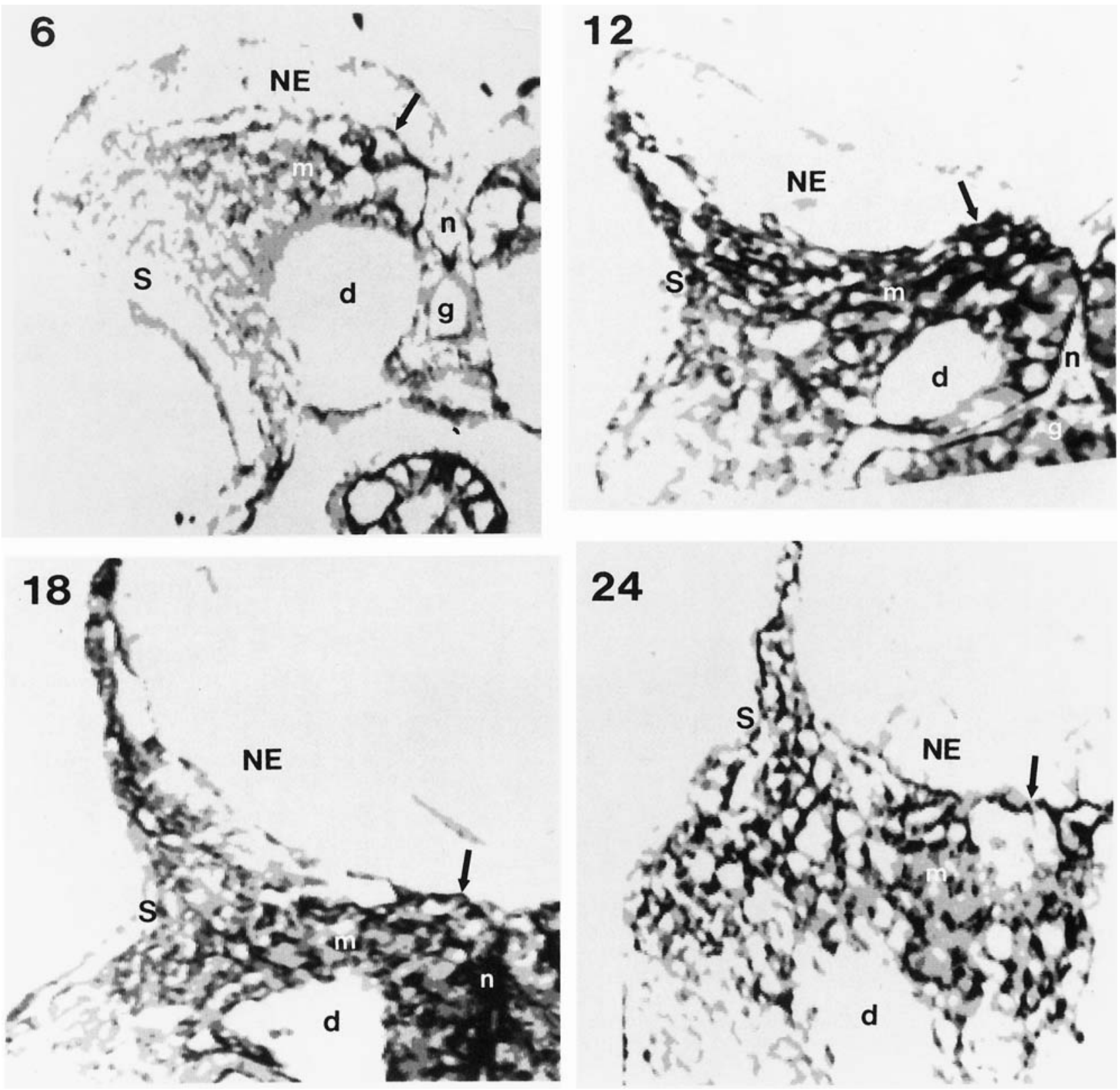

Figs. 1, 2. Representative difference picture images (DPI) showing the distribution of hyaluronate within control (Fig. 1) and DONtreated (Fig. 2) neural folds after 6, 12, 18, and $24 \mathrm{hr}$ of culture. Patterns were enhanced using a thresholding function to produce images with the six gray level steps, 0 (black) to 255 (white), shown in the legend in this and subsequent figures. $\times 225$.

Fig. 1. In control folds, the relative concentration of hyaluronate increased in the fold mesenchyme between 6 and $12 \mathrm{hr}$ of culture. At $18 \mathrm{hr}$, HA content was decreased in the central mesenchyme, but high concentrations were maintained in medial and lateral mesenchyme regions. At $24 \mathrm{hr}$, HA content was considerably decreased in both the central and medial mesenchyme. At all culture times the hyaluronate

content of the $\mathrm{NE} / \mathrm{Bm}$ (arrows) was greater in the median neural groove and its rim than in the central and lateral fold.

Fig. 2. In DON-treated folds, the relative concentration of hyaluronate increased only slightly, if at all, between 6 and $12 \mathrm{hr}$ of culture. At $12 \mathrm{hr}$, hyaluronate distribution in treated folds differed from that in controls in that medial regions of the mesenchyme were poorly stained and stain accumulated in the mesenchymal compartment formed by the junction of the neuroepithelium and surface ectoderm. This same pattern of hyaluronate distribution is observed at $18 \mathrm{hr}$ of culture. By $24 \mathrm{hr}$, the dorsomedial mesenchyme becomes more intensely stained. 

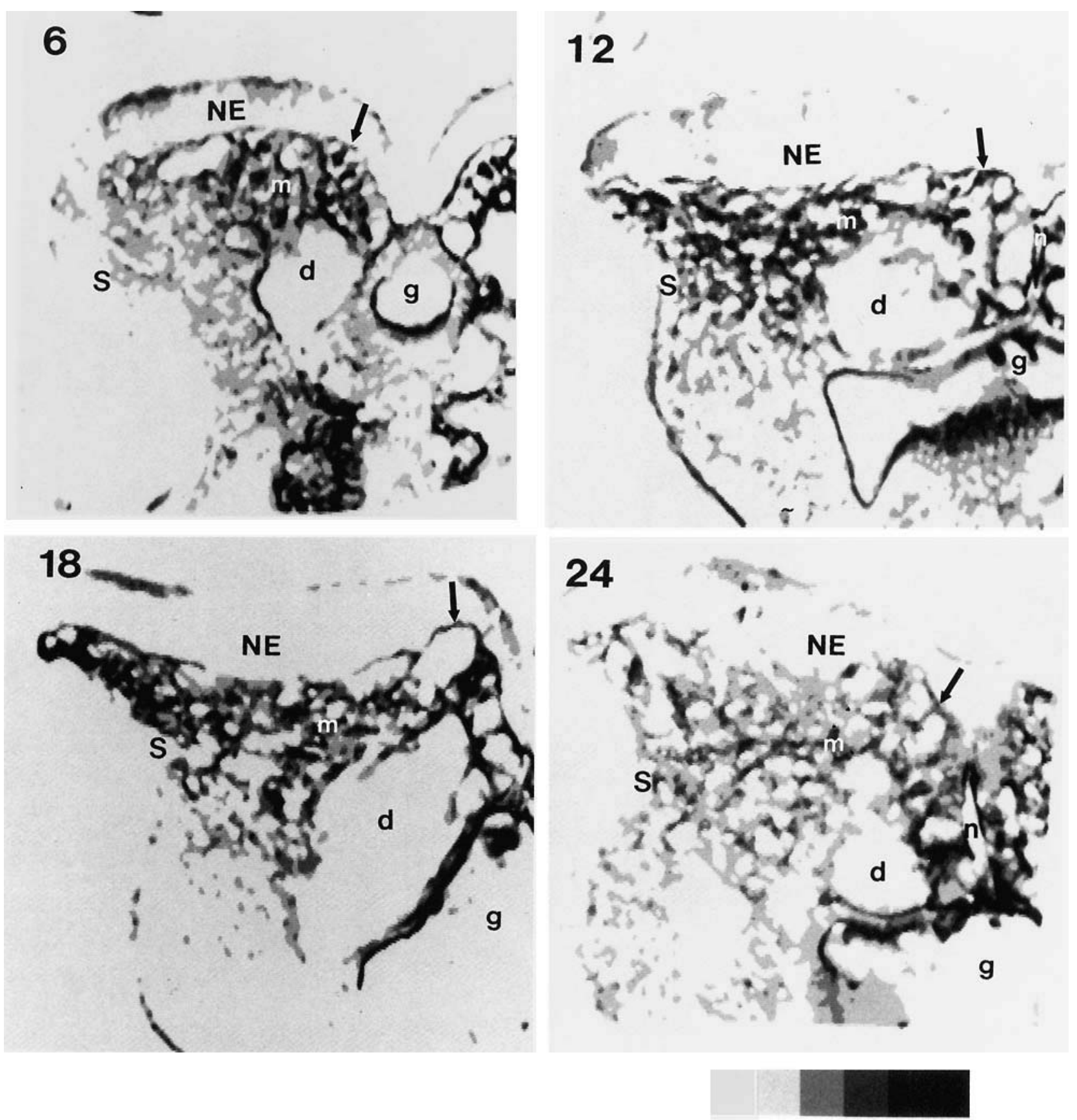

Fig. 2.

at $6 \mathrm{hr}$. By contrast, the pattern of label distribution in the neuroepithelial and surface ectodermal layers, as well as in the $\mathrm{NE} / \mathrm{Bm}$, is unchanged from that observed at $6 \mathrm{hr}$, although the relative amount of ${ }^{3} \mathrm{H}$-glucosamine incorporated is decreased. The pattern of label distribution in the $\mathrm{SE} / \mathrm{Bm}$ differs from that at $6 \mathrm{hr}$ in that it is now more intensely labeled dorsal to the first arch, where the neural fold narrows in width.

When ${ }^{3} \mathrm{H}$-glucosamine was added to the culture media at $16 \mathrm{hr}$, partially elevated neural folds of the con- trol embryos were often collapsed or everted by $18 \mathrm{hr}$. The anterior folds were the most sensitive to glucosamine addition, although the mesencephalic folds were affected as well. Only embryos that appeared normal macroscopically were analyzed. At $18 \mathrm{hr}$ the amount of ${ }^{3} \mathrm{H}$-glucosamine incorporated into the $\mathrm{NE}$, $\mathrm{SE}$, and mesenchyme is greatly diminished (Fig. 3). Only the more medial portions of the NE/Bm display intense labeling. However, the pattern of label distribution is unchanged from that observed at $12 \mathrm{hr}$. The 
6
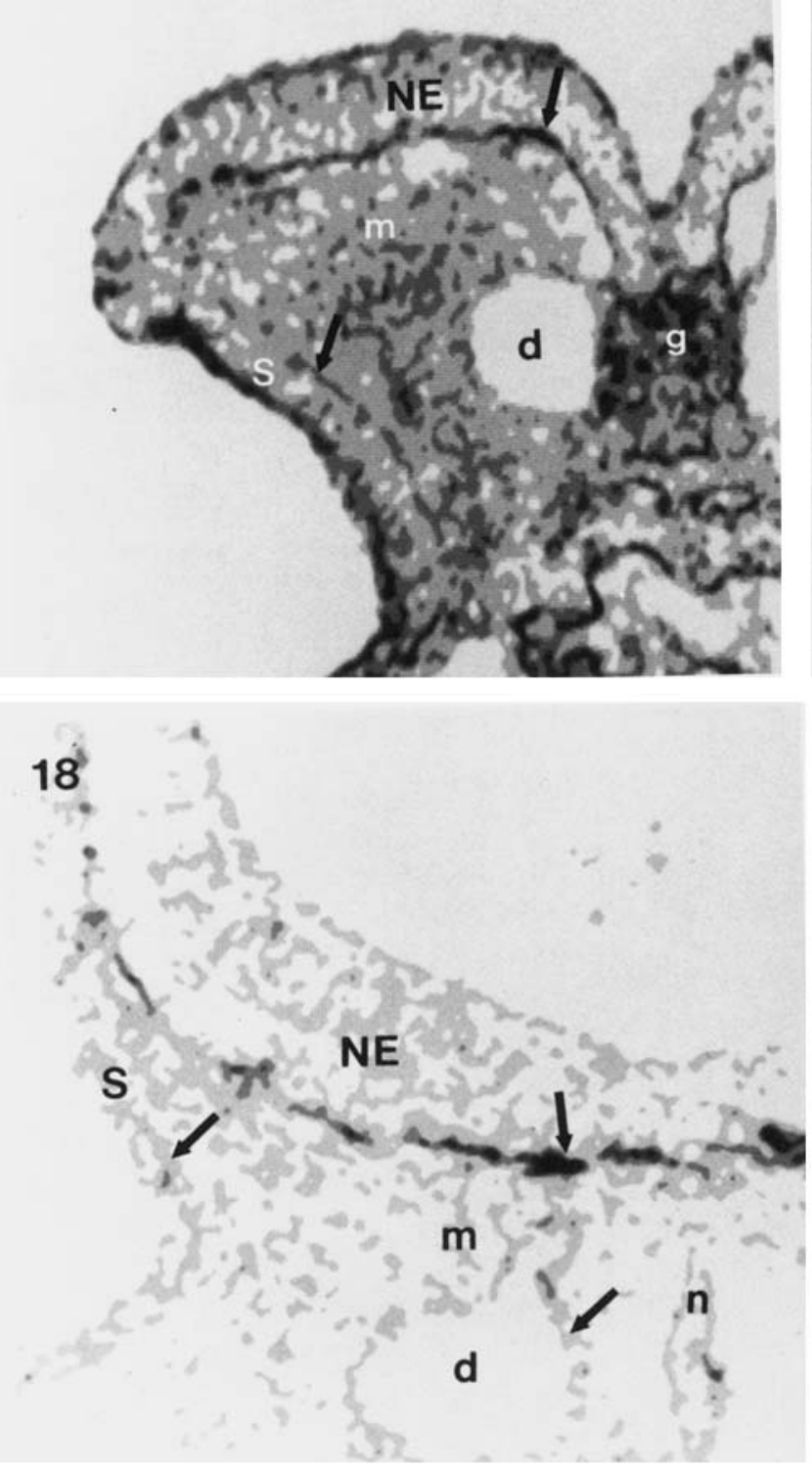
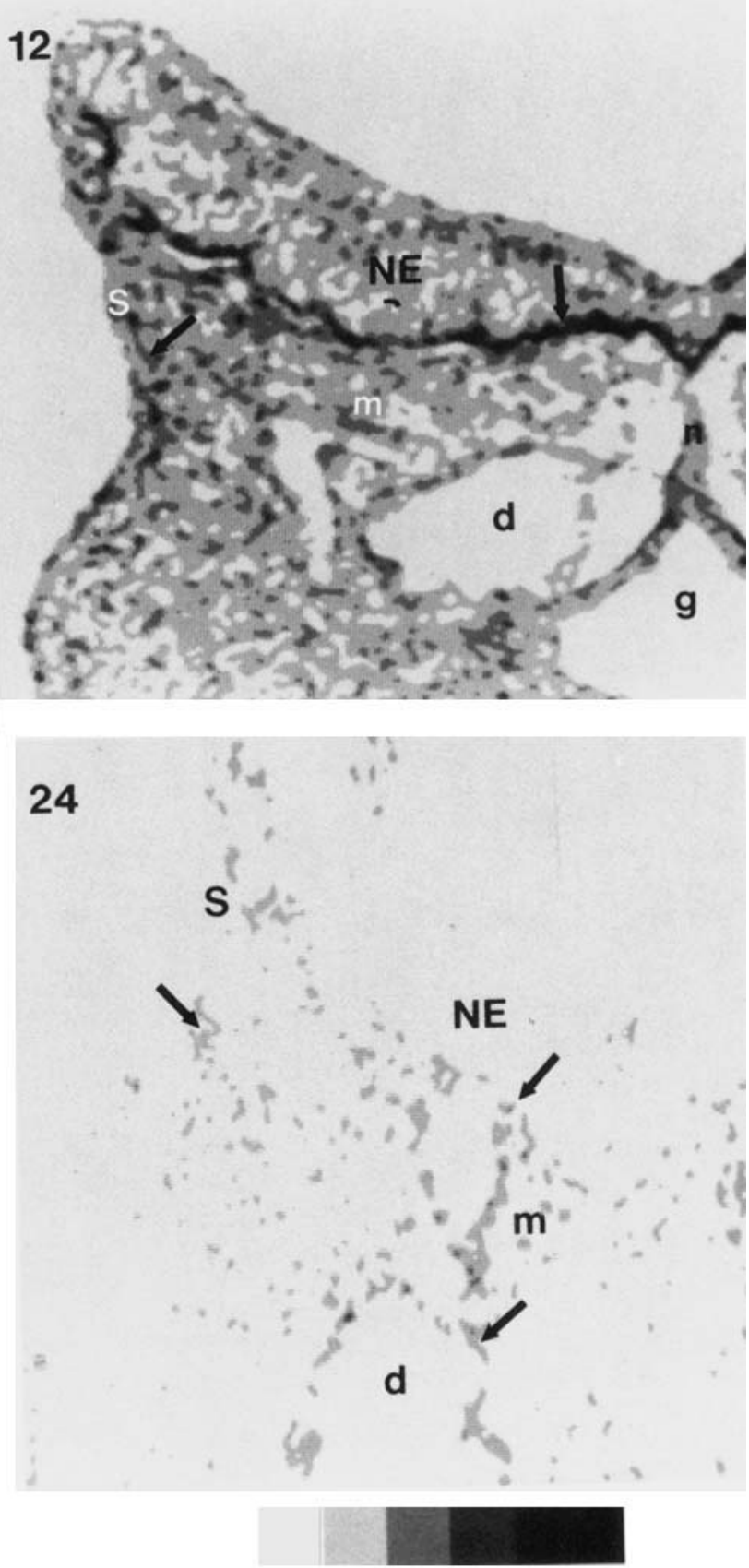

ithelial basement membrane and surface ectoderm remain well labeled. At $24 \mathrm{hr}$, very little label is incorporated; most of the label is associated with the basement membrane (arrows) of blood vessels.

Fig. 4. The pattern of ${ }^{3} \mathrm{H}$-glucosamine labeling in DON-treated folds at $6 \mathrm{hr}$ of culture is similar to that observed in control folds. At later culture times, DON-treated folds are much more heavily labeled than control folds although the labeling patterns at 12 and $18 \mathrm{hr}$ are similar to those observed at $12 \mathrm{hr}$ in controls. At $24 \mathrm{hr}$, more medial mesenchymal regions of DON-treated folds which were formerly devoid of ${ }^{3} \mathrm{H}$-glucosamine uptake are now labeled. Arrows indicate basement membranes. 

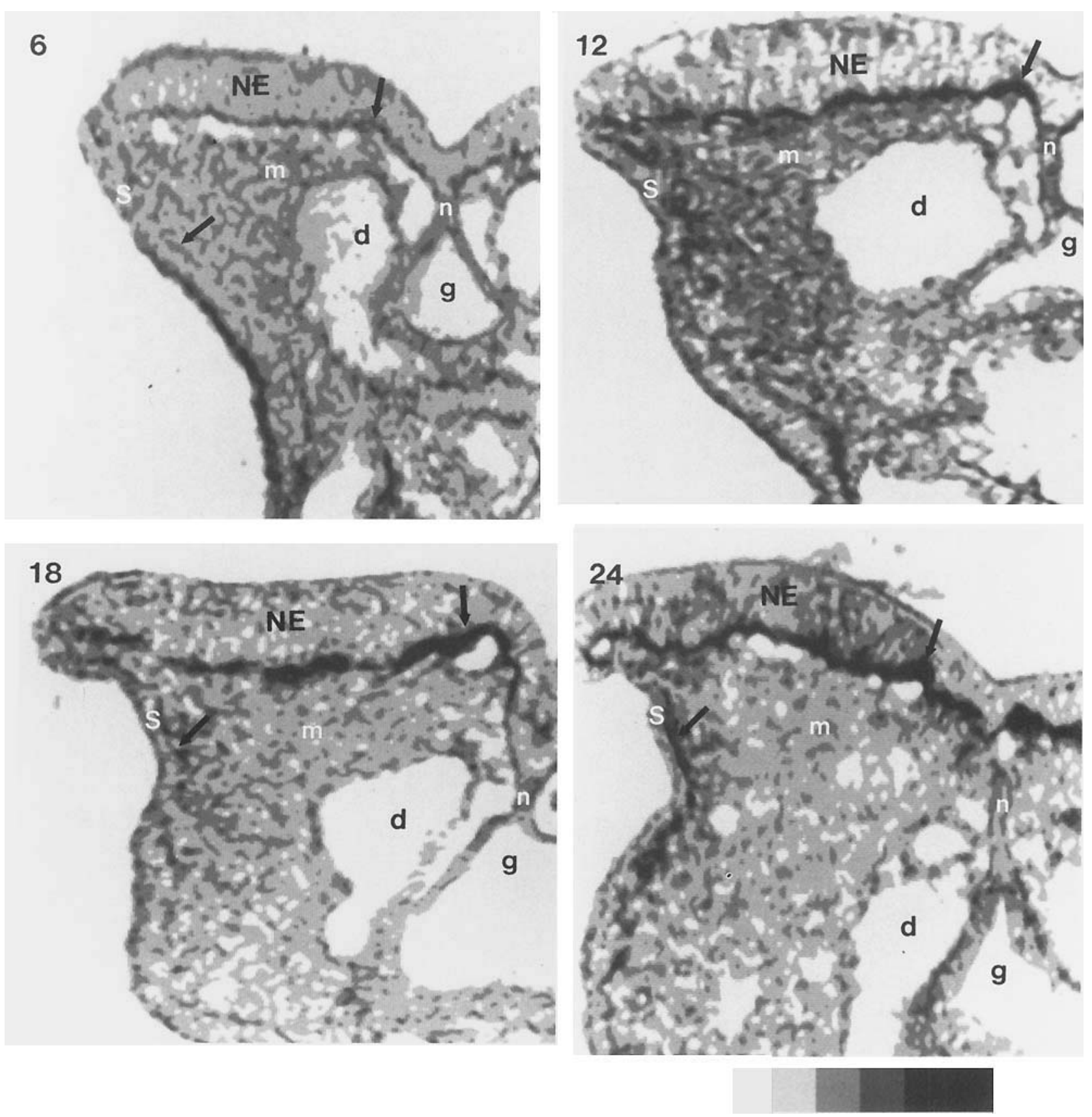

Fig. 4 .

dorsolateral mesenchyme supporting the elevating fold edge remains more densely labeled than central and medial mesenchymal regions. The major portion of the label associated with these latter regions is observed to encircle blood vessels. At $24 \mathrm{hr}$ of culture, very little ${ }^{3} \mathrm{H}$-glucosamine is incorporated into components of the mesenchyme extracellular matrix. Only the ECM encircling the dorsal aorta is distinctly labeled. Even the $\mathrm{NE} / \mathrm{Bm}$ that was intensely labeled at earlier ages has a discontinuous, patchy labeling pattern. No label is as- sociated with the neuroepithelium or the surface ectoderm.

\section{DON-treated embryos}

At $6 \mathrm{hr}$ of culture (Fig. 4), there are no apparenta differences in the patterns of label distribution between control and DON-treated mesenchyme and neural folds. At 12 hr of culture, the mesenchyme, NE, and $\mathrm{SE}$ of DON-treated embryos incorporate significantly more ${ }^{3} \mathrm{H}$-glucosamine than do their control counter- 
6

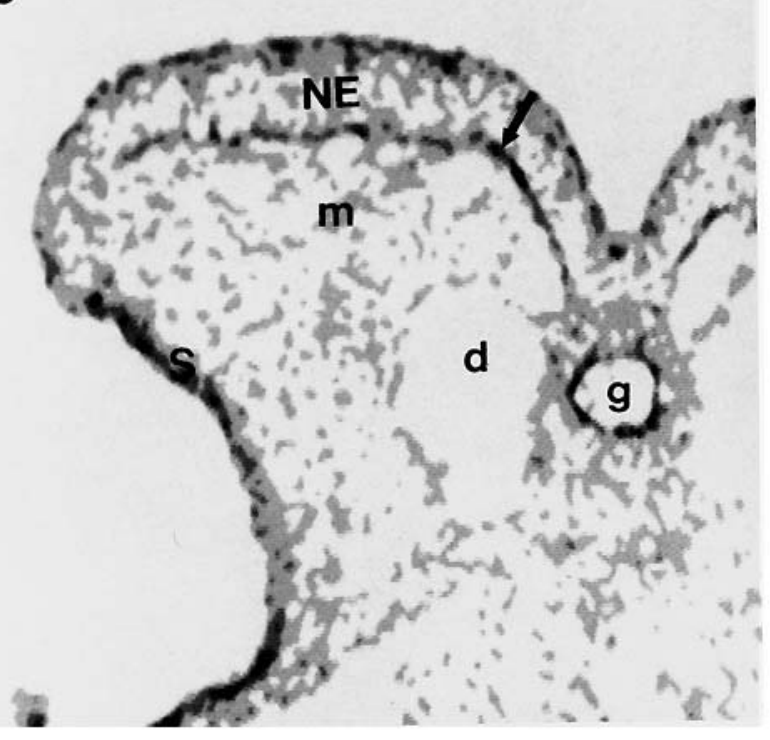

18

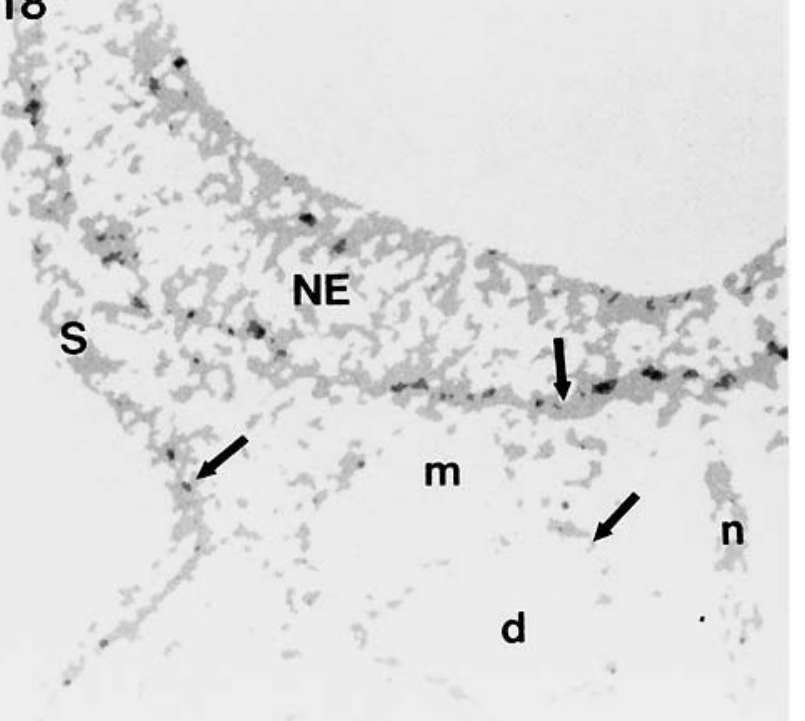

12

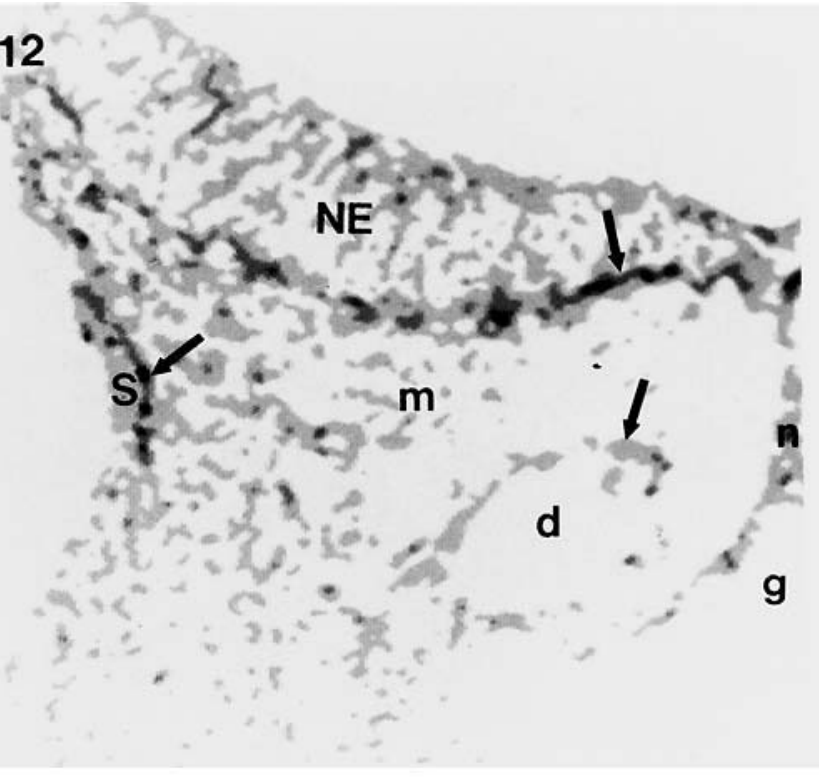

24<smiles>CCCCCCCCCCC</smiles>
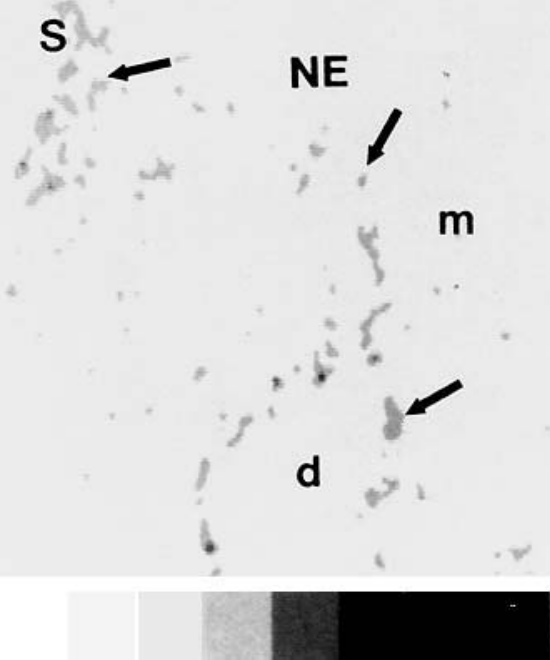

Fig. 5,6. Representative digital images of the distribution of ${ }^{3} \mathrm{H}$ glucosamine label corresponding to Streptomyces-hyaluronidase-resistant material in the mesencephalic neural folds of control (Fig. 5) and DON-treated (Fig. 6) embryos. $\times 225$.

Fig. 5. In control folds, most of the ${ }^{3} \mathrm{H}$-glucosamine label is removed by enzyme digestion. Some label remains in the lateral fold mesenchyme; most of the label associated with the central mesenchyme is

parts. However, the patterns of label distribution in treated embryos do not differ significantly from those of controls. As in the controls, in treated mesenchyme the lateral region is the most heavily labeled region; the medial mesenchyme adjacent to the median neural

removed. Label is also associated with the basement membranes (arrows) of the neuroepithelium, surface ectoderm, and blood vessels.

Fig. 6. (facing page) As in control folds, in DON-treated folds much of the label is removed by enzyme digestion. The remaining label is associated with basement membranes (arrows) and with the lateral mesenchyme. Label localized to the central fold mesenchyme is mostly removed by enzyme digestion.

groove is relatively label-free. The neuroepithelial basement membrane is intensely labeled, particularly in its more medial portions. However, unlike in the controls, in DON-treated embryos the basement membranes of the SE and NE enclosing the dorsolateral fold 

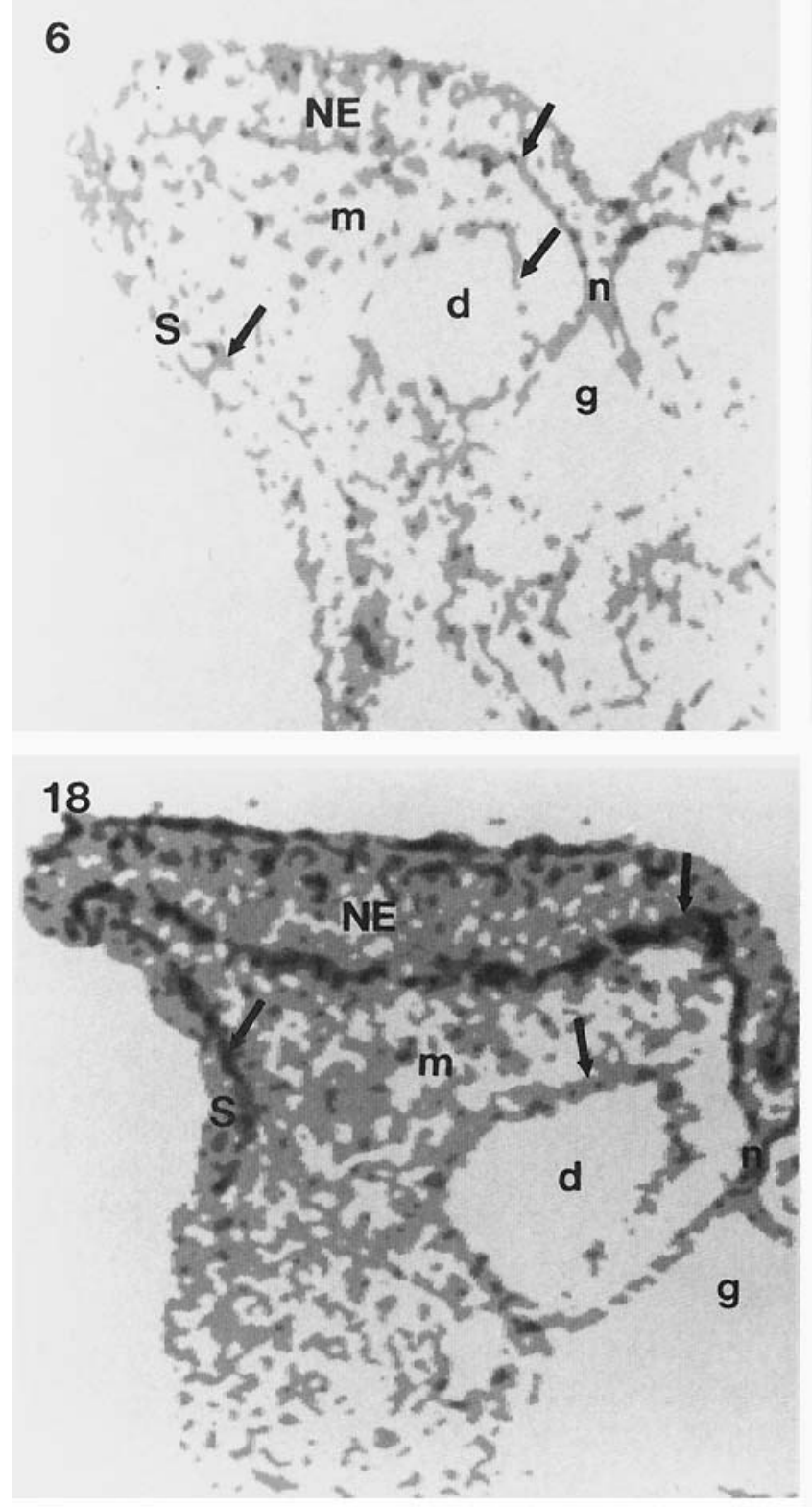
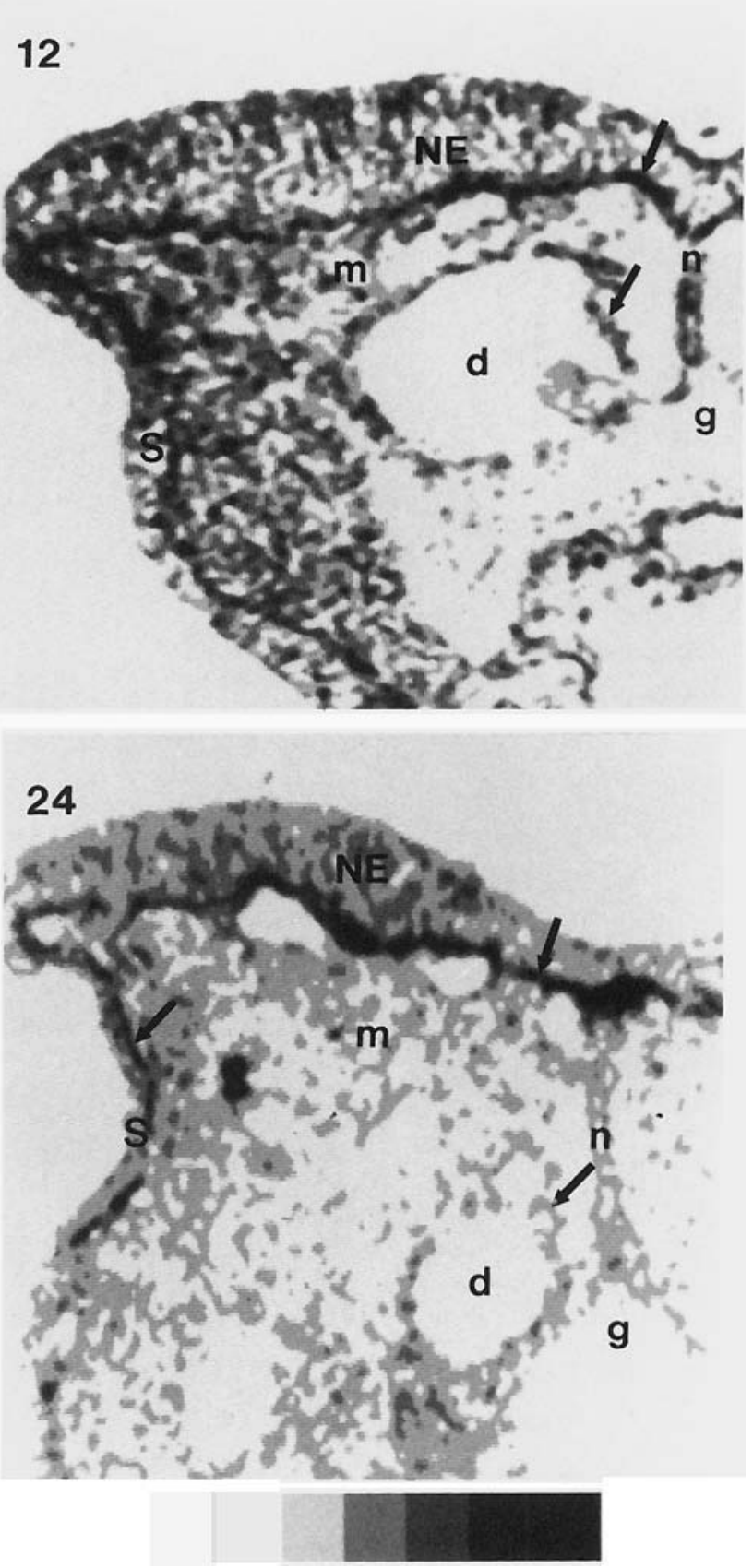

Fig. 6.

are associated with intense labeling. The pattern of label distribution in treated embryos remains unchanged at $18 \mathrm{hr}$, although the amount of ${ }^{3} \mathrm{H}$-glucosamine incorporated is decreased (Fig. 4). DON-treated folds and associated mesenchyme, however, are still much more intensely labeled than their control counterparts at $18 \mathrm{hr}$. At $24 \mathrm{hr}$, the amount of label incorporated increases, but the pattern of label distribution differs from that observed at earlier culture periods only in that label extends into the medial mesenchymal region that had been label free.

\section{Patterns of ${ }^{3} \mathrm{H}$-Glucosamine Incorporation after Streptomyces-Hyaluronidase Digestion}

\section{Controls}

Digestion of sections with Streptomyces hyaluronidase (SH) at $6 \mathrm{hr}$ of culture resulted in a profound decrease in the total amount of label in the neural folds (Fig. 5). SH digestion reduced the label intensity in all fold regions and in all components-the NE, SE, and adjacent mesenchyme. Some label is retained by the basement membranes of the neuroepithelium and sur- 

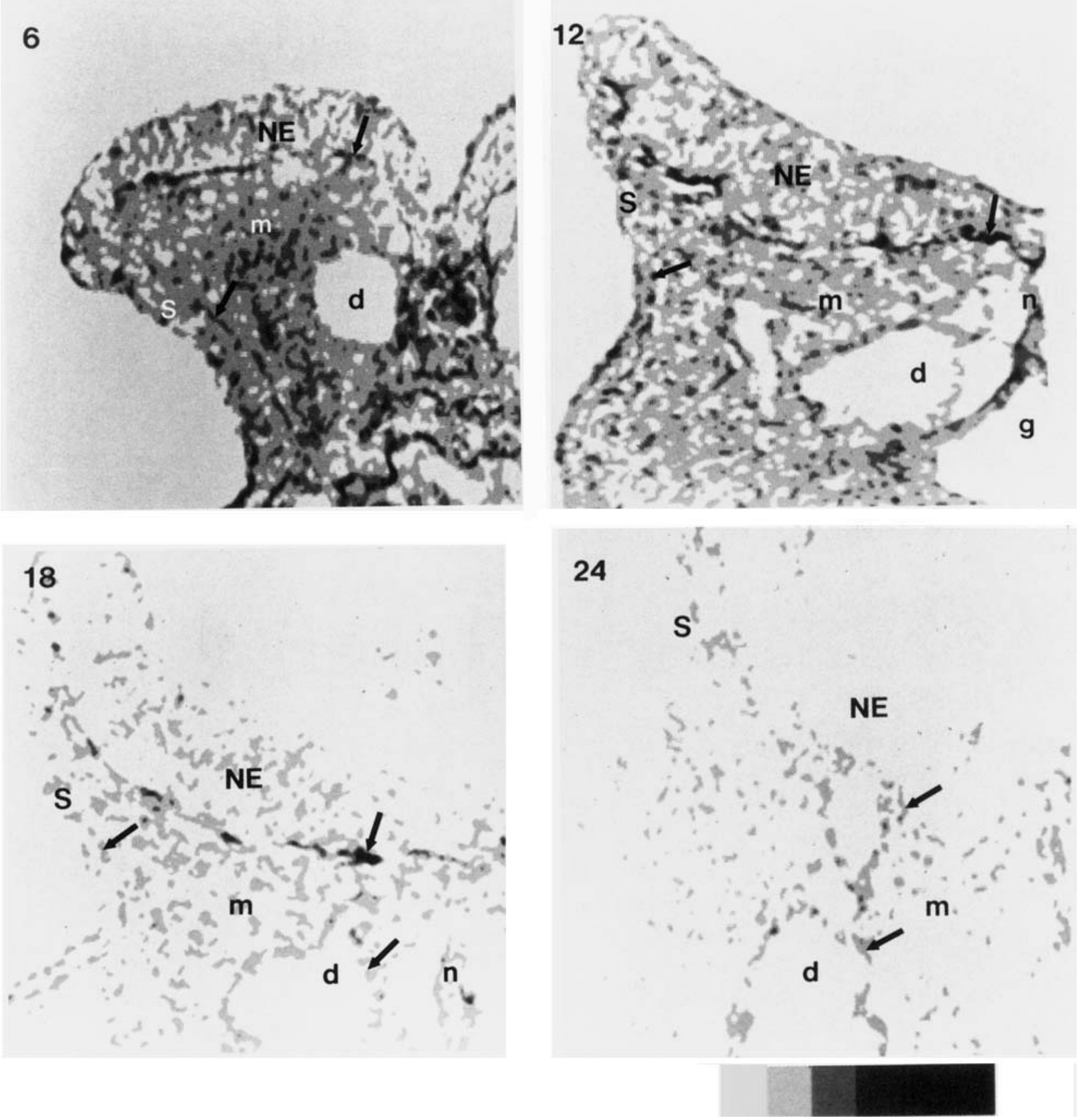

Figs. 7,8. Representative difference picture images (DPIs) showing the distribution of ${ }^{3} \mathrm{H}$-glucosamine-labeled $\mathrm{HA}$ within the mesencephalic neural folds of control (Fig. 7) and DON-treated (Fig. 8) embryos. $\times 225$.

Fig. 7. The patterns of labeled $\mathrm{HA}$ at $6 \mathrm{hr}$ are similar to the patterns of total label distribution. At 12 and $18 \mathrm{hr}$, labeled $\mathrm{HA}$ is distributed uniformly throughout the cranial mesenchyme and associated with basement membranes (arrows). At $24 \mathrm{hr}$, most of the labeled HA isassociated with the basement membranes of blood vessels.

Fig. 8. (facing page) Patterns of labeled HA at $6 \mathrm{hr}$ in DON-treated folds are similar to those in control folds. At 12 and $18 \mathrm{hr}$, much more ${ }^{3} \mathrm{H}$-glucosamine is incorporated into HA in DON-treated folds, but labeling patterns resemble those observed in control folds. At $24 \mathrm{hr}$ of culture, labeled HA is localized to the dorsomedial mesenchyme adjacent to the median neural groove which was devoid of label at earlier culture times. (Arrows) Basement membranes.

face ectoderm and by the apices of neuroepithelial cells, but little label remains in the mesenchyme. Only the label associated with the dorsal aorta and with the

mesenchymal compartment enclosed by the NE/SE junction survives digestion intact. At $12 \mathrm{hr}$, SH digestion again removes most of the ${ }^{3} \mathrm{H}$-glucosamine incor- 

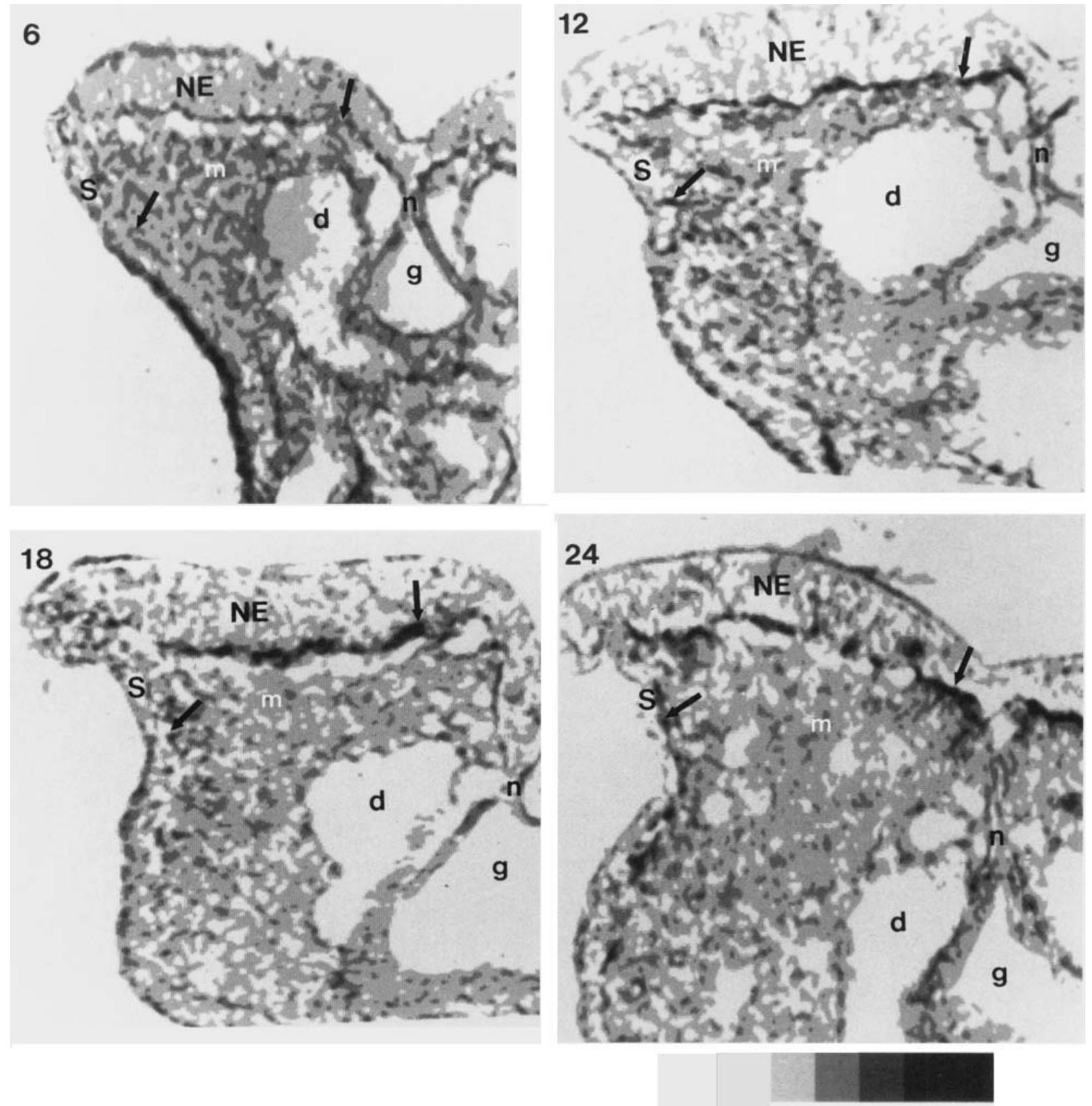

Fig. 8.

porated into the ECM of the central and medial mesenchyme, except for that associated with the dorsal aorta. However, in the lateral mesenchyme supporting the dorsolateral elevating fold edge, much of the label is retained. The $\mathrm{NE}$ and $\mathrm{SE}$ basement membranes, the $\mathrm{SE}$, and the apical surfaces of the NE also remain labeled. Once established at $12 \mathrm{hr}$, this pattern of SHresistant label distribution is unchanged at 18 and $24 \mathrm{hr}$ of culture (Fig. 5).

\section{DON-treated embryos}

The $\mathrm{SH}$ digestion of folds cultured in the presence of DON for $6 \mathrm{hr}$ results in a pattern of label distribution similar to that observed in digested control folds at $6 \mathrm{hr}$ (Fig. 6). Likewise, the label distribution pattern observed at $12 \mathrm{hr}$ does not differ significantly from that observed in controls, although much more label remains in the treated folds after digestion. Grain den- 
Table 1. Percent mesenchymal area covered by grains representing ${ }^{3}$ H-glucosamine-labeled HA

\begin{tabular}{lcc}
\hline Culture time (hr) & Control $^{1}$ & DON-treated $^{1}$ \\
\hline 6 & $0.4513 \pm 0.016$ & $0.4404 \pm 0.056$ \\
& $(\mathrm{n}=5)$ & $(\mathrm{n}=5)$ \\
12 & $0.3088 \pm 0.011^{*}$ & $0.3920 \pm 0.057^{\ddagger}$ \\
& $(\mathrm{n}=5)$ & $(\mathrm{n}=6)$ \\
18 & $0.1412 \pm 0.052^{*}$ & $0.3080 \pm 0.035^{*+}$ \\
& $(\mathrm{n}=3)$ & $(\mathrm{n}=6)$ \\
24 & $0.2076 \pm 0.039$ & $0.3692 \pm 0.075^{*}$ \\
& $(\mathrm{n}=5)$ & $(\mathrm{n}=6)$ \\
\hline
\end{tabular}

${ }^{1}$ Mean \pm standard deviation.

*Mean is significantly different $(P \leq 0.01)$ from that of preceding stage by analysis of variance.

A significant difference $(P<0.01)$ between DON-treated and controls of the same culture time.

sity is greatly reduced in the central and medial mesenchyme, as it is in controls, whereas the lateral mesenchyme remains intensely labeled. Label associated with the NE, SE, and their basement membranes is only slightly diminished by digestion. This pattern is maintained at 18 and $24 \mathrm{hr}$ of culture.

\section{Patterns of ${ }^{3} \mathrm{H}$-Glucosamine Incorporation in Difference Picture Images}

To evaluate the distribution of ${ }^{3} \mathrm{H}$-glucosamine-labeled HA within the neural fold during elevation, we analyzed patterns of label distribution in difference picture images (DPI), which were produced by the subtraction of the digitized image of a $\mathrm{SH}$-digested, ${ }^{3} \mathrm{H}$ glucosamine-labeled section from a similar image of an undigested, labeled section. Binary images were created from DPIs and used to calculate the percent of the total mesenchymal area occupied by ${ }^{3} \mathrm{H}$-glucosaminelabelled HA as a measure of total HA content of the fold (Table 1).

\section{Controls}

Because at $6 \mathrm{hr}$ of culture most of the ${ }^{3} \mathrm{H}$-glucosamine incorporated is removed by $\mathrm{SH}$ digestion, regardless of the region, the pattern of distribution of label attributable to HA in control folds at $6 \mathrm{hr}$ (Fig. 7) is not significantly different from that observed for total label. At $12 \mathrm{hr}$, the label incorporated into HA is observed to be evenly distributed throughout all regions of the fold cranial mesenchyme, although labeling density may be slightly greater in more lateral regions. The pattern of distribution of labeled HA in the NE and SE and their basement membrane is similar to that of total label in these fold components at $12 \mathrm{hr}$ (cf. Fig. 3). Only the amount of label is reduced. This pattern of label distribution is maintained at $18 \mathrm{hr}$, although the amount of ${ }^{3} \mathrm{H}$-glucosamine incorporated into HA decreases significantly (Table 1). The decrease in label in the central mesenchyme serves to accentuate the labeled HA encircling the dorsal aorta. At $24 \mathrm{hr}$, the major portion of labeled HA is associated with fold vessels. Very little label is incorporated into HA of the mesenchyme or basement membrane (Fig. 7).

\section{DON-treated embryos}

At $6 \mathrm{hr}$, there are no discernible differences between DON-treated and control folds in the amount of ${ }^{3} \mathrm{H}$ - glucosamine incorporated into HA (Table 1) or in the patterns of incorporation (Fig. 8). Although the amount of labeled HA within the mesenchyme decreases in the control folds at $12 \mathrm{hr}$ (Table 1), there is no significant decrease in labeled HA in the mesenchyme of treated folds. The labeling pattern in treated folds at $12 \mathrm{hr}$ is similar to that observed in control folds with one exception. At $12 \mathrm{hr}$ of culture, the amount of labeled $\mathrm{HA}$ associated with the $\mathrm{NE}$ relative to that in the mesenchyme is decreased with DON incubation. At $18 \mathrm{hr}$ of culture the total amount of labeled HA is decreased in the mesenchyme of treated folds (Table 1); however, the pattern of label distribution established at $12 \mathrm{hr}$ is maintained (Fig. 8). At $24 \mathrm{hr},{ }^{3} \mathrm{H}$-glucosamine-labeled HA becomes associated with regions of the medial mesenchyme adjacent to the median neural groove that are devoid of label at earlier culture times. This region and the dorsolateral and ventrolateral mesenchyme are regions of increased ${ }^{3} \mathrm{H}$-glucosamine incorporation into $\mathrm{HA}$.

\section{Ultrastructural Evaluation of DON-Treated Folds}

Neural folds cultured in the presence of DON were examined ultrastructurally to determine if any specific component of the extracellular matrix was removed or altered by DON treatment. At $8 \mathrm{hr}$ of culture, there are no apparent ultrastructural differences between the DON-treated and control folds. At 16 and $24 \mathrm{hr}$ of culture, however, treated folds can be easily distinguished from controls. The most prominent abnormalities are observed in the morphology of the neuroepithelial basal lamina (NE/Bl) (Fig. 9a,b). In DON-treated folds, some portions of the $\mathrm{NE} / \mathrm{Bl}$ appear to be duplicated (Fig. 9b). A characteristic basal lamina is absent from other regions, particularly in the central fold. The relative amounts of material precipitated by the tannic acid fixation also appear increased in the mesenchyme of treated folds. This is especially true in the sublaminar region, which is often the site of aggregates of tannic acid precipitate in treated folds.

In control folds after tannic acid fixation three components of the ECM are observed: 20 - to $35-\mathrm{nm}$ granules, 3- to 5-nm filaments, and 15- to 25-nm fibrils (Fig. $9 a)$. These three ECM components are present in treated folds (Fig. 9b,c), but in different proportions. The 20- to 35-nm granules compose a larger percentage of the ECM in treated folds, whereas the 3- to $5-\mathrm{nm}$ filaments are fewer in number and are complexed with the 20 - to $35-\mathrm{nm}$ granules to form large aggregates. Fibrils 15 to $25 \mathrm{~nm}$ in diameter are rare and are always complexed with granules in treated folds. Granules 30 to $60 \mathrm{~nm}$ in diameter, which normally are found within blood vessels and are probably components of the serum, are also found in the mesenchyme of treated folds. In DON-treated folds, the endothelia of the vessels subjacent to the NE do not form normal junctional complexes, allowing the seepage of serum into the mesenchymal compartment.

\section{DISCUSSION}

In this study we have analyzed patterns of ${ }^{3} \mathrm{H}$-glucosamine incorporation and hyaluronate (HA) distribution in the cranial mesenchyme during the elevation and convergence of the mouse mesencephalic neural folds using computer-generated difference picture im- 

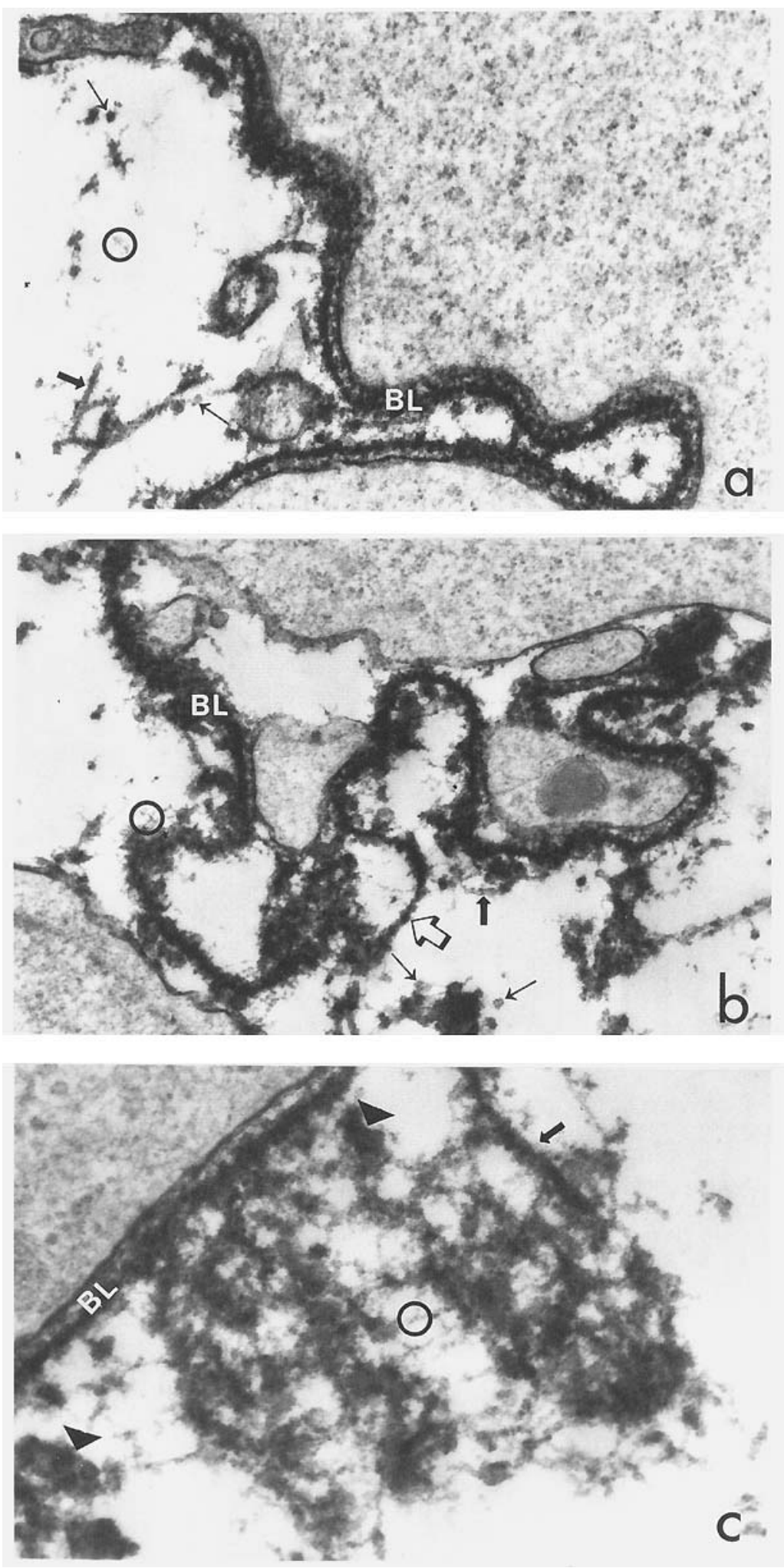

Fig. 9. Electron micrographs of the region immediately subjacent to the neuroepithelium of the central fold in control (a) and DON-treated (b,c) embryos after $24 \mathrm{hr}$ of culture. Unlike in control folds, where a continuous basal lamina (BL) lines the basal surface of neuroepithelial cells (a), in DON-treated folds the neuroepithelial basal lamina often encases cell processes and may have breaks or duplications (open arrow in b). An increased amount of tannic-acid-precipitable material in the sublaminar region is also associated with DON treat- ment. In both DON-treated and control folds the sublaminar extracellular matrix is composed of three components: 20- to 35-nm granules (thin arrows); 3- to 5-nm filaments (encircled); and 15- to 25-nm fibrils (thick arrows). In DON-treated folds, 20 - to $35-\mathrm{nm}$ granules constitute a larger percentage of the matrix. Three- to 5-nm filaments are fewer in number and are complexed with 20- to $35-\mathrm{nm}$ granules to form large aggregates (arrowheads). Fifteen- to 25-nm filaments are rare. a, $\times 32,500 ; \mathbf{b}, \times 35,000 ; \mathbf{c}, \times 60,000$. 
ages (DPI). A DPI is produced by the subtraction of the digitized images of Alcian blue-stained or ${ }^{3} \mathrm{H}$-glucosamine-labeled adjacent sections, one of which has been digested with an enzyme to specifically remove $\mathrm{HA}$. Thus, a DPI is an accurate depiction of the Alcian blue stain or the ${ }^{3} \mathrm{H}$-glucosamine label that is attributable to HA.

Our results indicate that regional differences in $\mathrm{HA}$ distribution patterns are not produced by regional differences in $\mathrm{HA}$ synthetic rates. Incorporation of ${ }^{3} \mathrm{H}$ glucosamine into $\mathrm{HA}$ in the cranial mesenchyme underlying the neural fold is elevated after $6 \mathrm{hr}$ of culture, but rapidly declines with further culture (Table 1). The distribution of ${ }^{3} \mathrm{H}$-glucosamine-labeled $\mathrm{HA}$ is uniform throughout the mesenchyme at 12,18 , and $24 \mathrm{hr}$ of culture. In contrast, Alcian blue-stained $\mathrm{HA}$ is fairly uniformly distributed throughout the mesenchyme at $12 \mathrm{hr}$ of culture, but at $18 \mathrm{hr}$, stain intensity is decreased in central regions of the mesenchyme. Stain intensity is maintained in the medial and lateral regions. At $24 \mathrm{hr}$, the relative amount of Alcian bluestained HA is decreased in the medial mesenchyme, but increased in the lateral mesenchyme. The central mesenchyme remains a region of relatively low HA concentration. These results suggest that HA is mainly synthesized in the mesenchyme before $12 \mathrm{hr}$ of culture when ${ }^{3} \mathrm{H}$-glucosamine incorporation into $\mathrm{HA}$ declines but Alcian blue staining reaches its peak intensity. These results also indicate that at 18 and $24 \mathrm{hr}$ of culture, patterns of Alcian blue staining are not determined by patterns of ${ }^{3} \mathrm{H}$-glucosamine incorporation.

Three possible explanations exist for the differences in the patterns of distribution of Alcian-blue-stained and ${ }^{3} \mathrm{H}$-glucosamine-labeled $\mathrm{HA}$. Because we know nothing about glucosamine pool sizes in the cranial mesenchyme of the elevating fold, we cannot rule out the possibility that patterns of ${ }^{3} \mathrm{H}$-glucosamine labeling do not reflect patterns of HA synthesis. An increased pool size at 18 and $24 \mathrm{hr}$ of culture may obscure patterns of ${ }^{3} \mathrm{H}$-glucosamine incorporation. We also have little information about HA turnover within the mesenchyme. The decrease in Alcian blue-stained HA in the central mesenchyme at $18 \mathrm{hr}$ and in the medial mesenchyme at $24 \mathrm{hr}$ could be the product of the increased degradation of $\mathrm{HA}$ in these regions. If this were the case, however, one would expect that unless other components were synthesized in increased quantities to stabilize the mesenchyme, the volume of the extracellular space would shrink. This shrinkage would be accompanied by an increase in mesenchymal cell density in the central mesenchyme. In this study the incorporation of ${ }^{3} \mathrm{H}$-glucosamine into all ECM components is decreased at 18 and $24 \mathrm{hr}$ of culture. In a companion study, we observed that at 18 and $24 \mathrm{hr}$ of culture there was a significant increase in the crosssectional area of the cranial mesenchyme (MorrisWiman and Brinkley, 1990b). This increased expansion of the mesenchyme was accompanied by a decrease in cell density in the central mesenchyme. The expansion of the mesenchyme in vivo has also been observed to be accompanied by a decrease in HA concentration and mesenchymal cell density in the central mesenchyme (Morris-Wiman and Brinkley, 1990a). These results suggest that the decrease in Alcian blue-stained $\mathrm{HA}$ at 18 and $24 \mathrm{hr}$ of culture is not produced by an increased degradation of $\mathrm{HA}$, but rather is the result of the expansion of the central and medial mesenchyme, decreasing the concentration of HA and separating resident mesenchymal cells.

The expansion of the central mesenchyme may result from the reorganization of the HA-rich extracellular matrix (ECM) in this region. HA occupies large molecular domains as a result of its highly charged nature (Comper and Laurent, 1978). At concentrations present in embryonic tissues, HA molecules intermesh with each other and with other matrix components, entrapping water and causing a high degree of hydration and an increase in volume (Toole, 1981). The expansion of an HA-rich ECM would be restricted by interactions between cells or other matrix components. If, however, these restraints were broken or altered, the HA meshwork would be allowed to expand, resulting in the sudden swelling of the tissue. Toole (1981) proposed that such a sequence of events leads to the sudden swelling of extracellular spaces which precedes neural crest cell migration, cushion cell invasion of the cardiac jelly, and mesenchymal invasion of the corneal stroma. We have previously proposed that expansion of the HArich matrix of the central fold mesenchyme is restrained by mesenchymal cell interactions and that the lateral expansion of the covering neuroepithelium causes the disruption of these interactions, allowing the HA meshwork to swell (Morris-Wiman and Brinkley, 1990a). The direction of the HA expansion is restricted by the attachment of the median neural groove to the notochord and by the lateral surface ectoderm. Analysis of patterns of distribution of Streptomyceshyaluronidase-insensitive ${ }^{3} \mathrm{H}$-glucosamine-labeled material reveals that at 12 and $18 \mathrm{hr}$ the mesenchyme of the lateral fold and the region subjacent to the neural groove becomes reinforced by non-HA matrix components. This reinforcement would serve as a buttress against the expansion of the central mesenchyme. Thus, expansion of the central mesenchyme pushes the folds medially resulting in their elevation. When the attachment between the median neural groove and notochord is disrupted at $24 \mathrm{hr}$, further expansion of the central and medial mesenchyme is directed dorsoventrally, increasing this dimension of the neural folds but not resulting in any further elevation.

If the forces that participate in the elevation of the neural folds are generated by the expansion of an HArich $\mathrm{ECM}$ in the central mesenchyme, then any disruption of the synthesis or organization of HA would interfere with the ability of the central mesenchyme ECM to expand and would result in failure of the neural folds to elevate. Diazo-oxo-norleucine (DON) is an agent that interferes with the synthesis of glycosaminoglycans (GAG) and the glycosylation of glycoproteins (GP) by competing with glutamine for aminotransferase in the synthesis of glucosamine (Ghosh et al., 1960). DON does not block the uptake of glucosamine (Telser et al., 1965). The neural folds of embryos cultured in the presence of DON fail to elevate (Sadler et al., 1980).

Patterns of distribution of Alcian blue-stained and ${ }^{3} \mathrm{H}$-glucosamine-labeled $\mathrm{HA}$ are abnormal in the cranial mesenchyme of DON-treated folds. The incorporation of ${ }^{3} \mathrm{H}$-glucosamine into $\mathrm{HA}$ is elevated in DONtreated mesenchyme above that in the control mesenchyme at all culture times except at $6 \mathrm{hr}$ (Table 
1). Not only is ${ }^{3} \mathrm{H}$-glucosamine incorporation into $\mathrm{HA}$ affected by DON treatment, but the amount of ${ }^{3} \mathrm{H}$-glucosamine incorporated into Streptomyces-hyaluronidase-resistant materials is also perturbed. The amount of labeled non-HA GAG and glycoproteins, relative to the amount of labeled HA, appears greatly increased in DON-treated folds, particularly at $12 \mathrm{hr}$ of culture. Increased ${ }^{3} \mathrm{H}$-glucosamine incorporation could be indicative of a DON-mediated increase in GAG and GP synthesis, but it is more likely the result of adding glucosamine to a DON-induced glucosamine-starved system. The decrease in Alcian blue-stained material in the mesenchyme, the $\mathrm{NE}$, and the $\mathrm{SE}$ of treated folds, as compared with controls, at 12 and $18 \mathrm{hr}$ also suggests that ECM synthesis is blocked by DON and that the increased incorporation is due to the glucosamine override. The patterns of ${ }^{3} \mathrm{H}$-glucosaminelabeled and Alcian blue-stained HA distribution in DON-treated mesenchyme are identical as 12,18 , and $24 \mathrm{hr}$ and are similar to those observed in control mesenchyme at $12 \mathrm{hr}$. This finding suggests that cells in treated mesenchyme retain their capability to synthesize GAG and will do so if the glucosamine block is removed. Patterns of HA distribution are determined by regional differences in HA synthesis. In controls at $12 \mathrm{hr}$, mesenchymal cells have produced the majority of their HA in the appropriate amount and location and are awaiting the initiation of matrix expansion; whereas in DON-treated folds at 12,18 , and $24 \mathrm{hr}$, the mesenchymal cells, although capable of synthesizing HA, cannot. Because the ECM of the mesenchyme lacks an adequate concentration of $\mathrm{HA}$, it cannot expand.

Alcian blue staining and ${ }^{3} \mathrm{H}$-glucosamine labeling patterns suggest that HA synthesis is in fact decreased in DON-treated mesenchyme. Present results, however, cannot rule out the possibility that the decrease in Alcian blue-stained material may be the result of an increased turnover of HA in the treated mesenchyme or an alteration in the organization of the HA in the ECM, which causes it to bind Alcian blue less effectively. The analysis of patterns of ${ }^{3} \mathrm{H}$-glucose incorporation into glucosamine in the mesenchyme of DONtreated and control embryos would have provided evidence in support of one of these alternatives. Unfortunately, the uptake of ${ }^{3} \mathrm{H}$-glucose by neurulating embryos proved to be prohibitively slow (unpublished observation).

Ultrastructural evidence suggests that the matrix synthesized in treated folds has an altered organization. The ECM of DON-treated folds is characterized by an increase in the number of 20- to $35-\mathrm{nm}$ granules, which are only rarely observed in control folds; a decrease in 3- to 5-nm filaments; and the appearance of $30-$ to $60-\mathrm{nm}$ granules. The 3- to 5-nm filaments are similar in appearance to filaments that have been determined by several investigators to be hyaluronate (Singley and Solursh, 1980; Markwald et al., 1979; Turley et al., 1985). The 20- to $35-\mathrm{nm}$ granules are reminiscent of chondroitin sulfate proteoglycan granules (CSPG) described by Turley et al. (1985). Similar granules predominate in the cardiac matrix of chick embryos in which HA is depleted by incubation with DON, but are rare in normal cardiac matrix (Markwald et al., 1978, 1979). One would suspect that interference with GAG and GP synthesis would not only have a quantitative effect, decreasing the total amount of ECM, but would also have a qualitative effect. The relative quantities of HA versus other ECM components are also altered by DON treatment, as demonstrated by the increased amount of Streptomyces-hyaluronidase-resistant labeled material in treated folds. Therefore, the normal organization of the ECM, as determined by interactions between components, would also be altered. The increase in CSPG-like granules and the decrease in 3- to 5-nm filaments in the ECM of neural folds and cardiac matrix with DON treatment may be the result of a relative deficiency in some other component, such as HA, which normally has a major role in orchestrating ECM organization.

\section{LITERATURE CITED}

Brinkley, L.L., and J. Morris-Wiman 1987 Computer-assisted analysis of hyaluronate distribution during morphogenesis of the mouse secondary palate. Development, 100:629-635.

Comper, W.D., and T.C. Laurent 1978 Physiological function of connective tissue polysaccharides. Physiol. Rev., 58:255-315.

Derby, M.A., and J.E. Pintar 1978 The histochemical specificity of Streptomyces hyaluronidase and chondroitinase ABC. Histochem J., 10:529-547.

Ghosh, S., H.J Blumenthal, E.A. Davidson, and S Roseman 1960 Glucosamine metabolism. V. Enzymatic synthesis of glucosamine-6-phosphate. J. Biol. Chem., 235:1265-1272.

Heifetz, A., W.J. Lennarz, B. Libbus, and Y. Hsu 1980 Synthesis of glycoconjugates during the development of mouse embryos in vitro. Dev. Biol., 80:398-408.

Markwald, R.R., T.P. Fitzharris, H. Bank, and D.H. Bernanke 1978 Structural analysis on the matrical organization of glycosaminoglycans in developing endocardial cushions. Dev. Biol., 62:292316

Markwald, R.R., F.M. Funderberg, and D.H. Bernanke 1979 Glycosaminoglycans: Potential determinants in cardiac morphogenesis. Texas Rept Biol. Med. 39:253-270.

Morriss, G.M., and M. Solursh 1978a Regional differences in mesenchymal cell morphology and glycosaminoglycans in early neuralfold stage rat embryos. J. Embryol. Exp. Morphol., 46:37-52.

Morriss, G.M., and M. Solursh $1978 \mathrm{~b}$ The role of the primary mesenchyme in normal and abnormal morphogenesis of mammalian neural folds. Zoon, 6:33-38.

Morris-Wiman, J., and L.L. Brinkley 1990a Changes in mesenchymal cell and hyaluronate distribution correlate with in vivo elevation of the mouse mesencephalic neural folds. Anat. Rec., in press.

Morris-Wiman, J., and L.L. Brinkley $1990 \mathrm{~b}$ The role of the mesenchyme in mouse neural fold elevation. I. In vitro patterns of mesenchymal cell proliferation. Am. J. Anat., (this issue).

Ohya, T., and Y. Kaneko 1970 Novel hyaluronidase from Streptomyces. Biochim. Biophys. Acta, 198:607-609.

O'Shea, K.S.O., K.K. Svoboda, and M.A. Webb 1982 Media variation in whole embryo culture. Teratology, 25:A65-A66.

Sadler, T.W., J. Langman, and D. Burke 1980 Mammalian embryos in culture: a unique approach to craniofacial research. In: Current Research Trends in Prenatal Craniofacial Development. R.M. Pratt and R.M. Christensen, eds. Elsevier North-Holland, Amsterdam, pp. 133-147.

Singley, C.T., and M. Solursh 1980 The use of tannic acid for the ultrastructural visualization of hyaluronic acid. Histochemistry, 65:93-102.

Solursh, M., and G.M. Morriss 1977 Glycosaminoglycan synthesis in rat embryos during the formation of the primary mesenchyme and neural folds. Dev. Biol., 57:75-86.

Steele, C.E., and D.A.T. New 1974 Serum variants causing the for mation of double hearts and other abnormalities in explanted rat embryos. J. Embryol. Exp. Morphol., 31:707-719.

Telser, A., H.C. Robinson, and A. Dorfman 1965 The biosynthesis of chondroitin-sulfate protein complex. Proc. Natl Acad. Sci. USA, 54:912-919

Toole, B.P. 1981 Glycosaminoglycans in morphogenesis. In: Cell Biology of Extracellular Matrix. E. Hay, ed. Plenum Press, New York, pp. 259-294.

Turley, E.A., C.A. Erickson, and R.P. Tucker 1985 The retention and ultrastructural appearances of various extracellular matrix molecules incorporated into three-dimensional hydrated collagen lattices. Dev. Biol., 109:347-369. 